\title{
LA RESPUESTA DE DIRECTORES ESCOLARES ANTE LAS POLÍTICAS DE INCLUSIÓN ESCOLAR EN CHILE
}

\author{
Marta Quiroga ${ }^{1}$ \\ Felipe Aravena ${ }^{2}$
}

\begin{abstract}
RESUMEN
Chile presenta uno de los sistemas educativos más segregados del mundo. La inclusión es una oportunidad para revertir este dramático escenario educativo. El presente estudio tiene por objetivo indagar cuáles serían las condiciones necesarias para la instalación de prácticas educativas inclusivas en establecimientos escolares chilenos, desde la perspectiva de siete directores escolares. Este estudio, a través de una metodología mixta, utilizando la técnica concept mapping y un análisis factorial de los datos, concluye que los recursos, la actitud personal y las políticas públicas son las tres variables más importantes que influyen en la instalación de prácticas inclusivas. Los resultados de esta investigación aportan al debate de la política pública nacional con información clave para los tomadores de decisión, con el fin de desplegar acciones tendientes a mitigar las resistencias que trae consigo el incluir a otros.
\end{abstract}

Conceptos clave: directores escolares, inclusión, liderazgo escolar.

\section{CHILEAN SCHOOL PRINCIPALS' RESPONSE TO PUBLIC POLICIES ON INCLUSION}

ABSTRACT

Chile presents one of the most segregated educational systems in the world. Inclusion is an opportunity to reverse this dramatic educational scenario. The purpose of this study is to investigate necessary conditions in order to effectively implement inclusive educational practices in Chilean schools, from the perspective of seven school principals. This study, through the application of a mixed methodology, the concept mapping technique and a factorial analysis of the data, concludes that resources, personal attitudes and public policies are the three most important variables that influence the implementation of inclusive practices within schools. The results of this research contribute to the national debate with key information for policymakers, in order to analyze actions, which tend to mitigate resistance towards including others.

Keyconcepts: educational leadership, inclusion, school principals.

1 Escuela de Pedagogía, Pontificia Universidad Católica de Valparaíso, Valparaíso, Chile. Contacto: marta.quiroga@pucv.cl

2 Centro de Liderazgo para la Mejora Escolar, Lideres Educativos, Pontificia Universidad Católica de Valparaíso, Valparaíso, Chile. Contacto: felipe.aravena@pucv.cl 


\section{Introducción}

Chile presenta uno de los sistemas educativos más segregados del mundo. La convergencia de factores históricos, financieros, políticos y socioculturales ha producido que esta segregación educativa aumente progresivamente a través de las últimas décadas (Hsieh \& Urquiola, 2006). La segregación escolar es un fenómeno comprensible a causa de la masificación y posterior profundización de políticas neoliberales de mercado en el campo educativo nacional (Drago y Paredes, 2011; Hsieh \& Urquiola, 2006; Levin, 2011). Esto ha sido denominado como el período de reformas neoliberales, que comprende desde 1990 hasta el año 2010 (Cavieres, 2011). En este tiempo, Chile asumió que la modernización del sistema educativo dependía de la introducción de políticas neoliberales (Aravena \& Quiroga, 2016; Mizala \& Torche, 2012); así el neoliberalismo fue entendido como sinónimo de modernización. Por efecto, la competencia y segregación se posicionaron como producto y prácticas resultantes de un modelo esencialmente neoliberal (Gauri, 1998; Mizala \& Romaguera, 1998).

Selección, competencia y homogeneización son conceptos clave para dimensionar el impacto de las políticas neoliberales en el sistema educacional chileno (Verger, Bonal y Zancajo, 2016). Así mismo, Sapelli (2003) explica que el sistema educativo nacional en su diseño estructural, denominado en la literatura internacional como Chilean voucher system, potencia prácticas excluyentes y poco inclusivas para y en las escuelas. Esto, porque la misma estructura separatista entre escuelas municipales, subvencionadas y particulares pagadas se hace semejante a la división de clases entre pobres, clase media y clase alta. Si bien esta segregación pareciera estar únicamente posicionada en términos socioeconómicos, impacta en una dimensión socioemocional, específicamente en lo que respecta a las oportunidades y aspiraciones de los estudiantes y sus familias (Drago \& Paredes, 2011; Mizala \& Torche, 2012; Villalobos y Valenzuela, 2012).

Para revertir este dramático panorama educativo, en los últimos años el Ministerio de Educación de Chile, Mineduc, ha impulsado diversas políticas de inclusión dentro de las escuelas. En 
términos prácticos, esto ha significado nuevas responsabilidades y funciones para los equipos directivos y, particularmente, para los directores escolares. La literatura nacional se ha preocupado de explorar este fenómeno, sin embargo, son escasos los estudios que investigan los retos que enfrentan los directores escolares chilenos cuando buscan instalar prácticas y políticas de inclusión dentro de sus escuelas. El presente estudio tiene por objetivo indagar cuáles serían las condiciones necesarias para la instalación de prácticas educativas inclusivas en los establecimientos escolares chilenos, desde la perspectiva de los directores escolares.

La relevancia de este estudio radica en la posibilidad de identificar qué es considerado importante para liderar y gestionar prácticas y políticas de inclusión dentro de las escuelas chilenas desde la perspectiva de los directivos docentes. Los resultados de esta investigación aportan al debate de la política pública nacional con información clave para los tomadores de decisión, con el fin de desplegar acciones tendientes a mitigar las resistencias que trae consigo el incluir a otros. Estas acciones pueden estar inicialmente asociadas con recursos financieros, sin embargo, también se encuentran vinculadas con el desarrollo de actividades formativas que promueven la inclusión en los futuros líderes escolares. Es una tarea pendiente en Chile favorecer el desarrollo de prácticas de liderazgo que tiendan al éxito de cada estudiante, mediante la implementación de acciones de desarrollo profesional para sus docentes y directivos, así como el fortalecimiento de las relaciones con las familias y comunidades. Este estudio pretende aportar indagando cuáles serían las condiciones necesarias para la instalación de prácticas educativas inclusivas en los establecimientos escolares en Chile, desde la perspectiva de los directivos docentes.

\section{Políticas de inclusión en Chile}

En los últimos años, las políticas públicas han delineado e integrado nuevos elementos a desarrollar dentro de las escuelas. Uno de esos es la comprensión conceptual y actitudinal de lo que significa la diversidad cultural, cognitiva y socioeconómica en la realidad contextual a nivel nacional (López, Julio, Morales, Rojas y Pérez, 2014). En términos 
más puntuales, Chile ha avanzado en la conceptualización desde prácticas de integración de la diversidad hacia prácticas de inclusión. Este progreso se refleja en la promulgación de la Ley no 20.845 de Inclusión y Fin del Lucro, publicada en el Diario Oficial de la República de Chile en junio de 2015, que establece en su artículo 1, letra K, que:

el sistema tenderá a eliminar todo tipo de discriminación arbitraria que impida el aprendizaje y la participación del estudiante. Asimismo, el sistema promoverá que los establecimientos educativos se conviertan en un lugar de encuentro entre los estudiantes de diferentes condiciones socioeconómicas, culturales, étnicas, de género, nacionales o religiosas.

Por lo tanto, se infiere que Chile espera construir escuelas inclusivas, abiertas al diálogo y al aprendizaje desde la diversidad. En la misma línea, el país ha focalizado sus esfuerzos en establecer lineamientos genéricos para impulsar prácticas y políticas de inclusión. Los reglamentos promulgados con anterioridad Decreto 83 y 170 - enfrentaron la inclusión desde el punto de vista del estudiante con necesidades educativas especiales (NEE) y la obligación de desarrollar la integración con financiamiento adicional para apoyar los procesos de aprendizaje. De esta forma, la integración se sostenía en problemáticas motoras, sensoriales y cognitivas, entre otras. En cambio, la Ley n 20.845 se alinea con la propuesta de la Unesco, que indica que "el propósito de la inclusión es dar respuestas apropiadas al amplio espectro de necesidades de aprendizaje, tanto en entornos formales como informales" (United Nations Educational, Scientific and Cultural Organization, Unesco, 2005, p. 14). Así, se ha establecido que el propósito de la educación inclusiva es permitir que los profesores y estudiantes se sientan cómodos frente a la diversidad y que no la perciban como un problema, sino como un reto y una oportunidad para enriquecer las formas de enseñanza y aprendizaje.

La educación inclusiva, entonces, tiene el objetivo de abordar y responder a la diversidad de los estudiantes. Echeita y Ainscow (2011) presentan cuatro elementos característicos que guían una política de inclusión: 
- La inclusión como un proceso, una búsqueda constante de mejores formas de responder a la diversidad de los estudiantes, apreciándola y usándola de manera positiva y que, como todo proceso, necesita tiempo para ser implementada.

- La inclusión busca la presencia, participación y éxito de cada estudiante, fomentando la diversidad sin tender a procesos de homogenización y/o discriminación educativa.

- Es necesario identificar y eliminar las barreras que impiden el ejercicio efectivo de los derechos de los estudiantes.

- Énfasis en aquellos grupos estudiantiles que se encuentran en riesgo de marginación, exclusión o fracaso escolar.

En suma, la inclusión se hace cargo de manera compleja y multidimensional de la diversidad que hoy es posible encontrar en las escuelas chilenas.

La perspectiva de la inclusión es particularmente importante en el caso chileno. Diversas investigaciones (García Huidobro, 2007; González, 2003; Villalobos y Valenzuela, 2012) han demostrado que las desigualdades económicas se reproducen en las escuelas a través del valor cobrado a los padres (matrículas, mensualidades, arancel, etc.), lo que ha provocado la segmentación del mercado educativo (Cavieres, 2011). La Ley n ${ }^{\circ} 20.845$ de Inclusión y Fin del Lucro busca terminar con esta condición de manera progresiva, reemplazando el copago de los padres por una contribución estatal y prohibiendo los procesos de selección de estudiantes. Estos cambios legislativos están produciendo diversas transformaciones en el sistema escolar, por lo que resulta fundamental analizar las condiciones necesarias para desarrollar estrategias de apoyo y aseguramiento de la ley en un ámbito educativo. Estos cambios traen aparejada una responsabilidad clave que desafía a los directores escolares cotidianamente en el desempeño de su rol.

Como se aprecia, Chile vive un cambio cualitativo en la concepción de diversidad, transitando desde las políticas de integración de estudiantes con impedimentos cognitivos, necesidades educativas especiales y con diferentes niveles de éxito académico (Cuadra, 2008; Fernández, 2012) hacia políticas de inclusión que 
complejizan aún más el escenario nacional. En términos de política pública esto ha sido difícil de implementar, en tanto que en la práctica, las escuelas y sus equipos directivos han debido sortear los obstáculos y desafíos que acarrea la inclusión. La evidencia empírica que presenta la literatura nacional aún es escasa.

\section{El liderazgo directivo y los directores en Chile}

El liderazgo es influencia, movilización y transformación. Más precisamente el liderazgo en educación puede ofrecer dirección, inspiración y motivación dentro de las escuelas (Hargreaves $\&$ Shirley, 2012), estableciendo las condiciones necesarias para desarrollar culturas de colaboración con el objetivo de mejorar e impactar en los aprendizajes de los estudiantes (Anderson, 2008; Leithwood, Harris $\&$ Hopkins, 2008). El liderazgo, como proceso de influencia y cambio, es ejercido primordialmente por los directores escolares y sus equipos, de ahí que la posibilidad de desarrollar equipos capaces, comprometidos y motivados para instalar y cambiar prácticas pedagógicas sea una tarea compleja y altamente demandante, que implica distribuir responsabilidades y liderazgos (Harris, 2009).

Existe abundante evidencia internacional que señala que el liderazgo educativo es la segunda variable que más impacta en el mejoramiento de los aprendizajes de los estudiantes (DarlingHammond, Meyerson, La Pointe \& Orr, 2010; Leithwood et al., 2008). Al mismo tiempo, son variados los estudios que expresan que los directores escolares pueden ejercer influencia en la modificación de las prácticas de enseñanza realizadas por los profesores (Anderson, 2008; Hargreaves \& Shirley, 2012; Leithwood et al., 2008). Esto explica, entonces, que los directores pueden influir en el aprendizaje y la enseñanza escolar. A nivel nacional, diversos estudios posicionan al liderazgo directivo como factor clave en la mejora y cambio escolar. Por ejemplo, Horn y Marfán (2010), a través de una revisión bibliográfica que analiza 14 estudios empíricos realizados en Chile, concluyeron que el liderazgo educativo incide como factor en el logro de aprendizajes de calidad. Por lo tanto, la teoría internacional también se confirma en el panorama nacional: el liderazgo educativo impacta de manera indirecta en los aprendizajes de los estudiantes 
(Anderson, 2008; Darling-Hammond et al., 2010; Hargreaves \& Shirley, 2012; Harris, 2009; Leithwood et al., 2008).

Recientes estudios nacionales buscan indagar en el rol y las prácticas de los directores en Chile (Donoso, Benavides, Cancino, Castro y López, 2011). En una primera aproximación, Weinstein y Muñoz (2014) investigaron las características de los directores chilenos en términos de género, tamaño de escuelas, edad y procedencia escolar, entre otros elementos relevantes. El aporte de este estudio permitió obtener una fotografía nacional respecto de quiénes ocupan el rol directivo dentro de las escuelas. En términos de prácticas directivas, un último estudio demostró que los directores presentan problemas para concentrar sus esfuerzos en convertirse en líderes pedagógicos debido a la multiplicidad de demandas externas que hacen difícil focalizar los procesos más allá de la esfera administrativa (Weinstein, Cuellar, Hernández y Fernández, 2016). No obstante, hoy es posible observar a directores escolares más empoderados, capaces de realizar cambios, confiados en marcar una diferencia significativa en las trayectorias escolares de sus organizaciones (Bellei, Muñoz, Pérez y Raczynski, 2004; Weinstein $\&$ Muñoz, 2014). Si bien los estudios en torno al rol y las prácticas de los directores en Chile han ido aumentando progresivamente, no es posible observar todavía estudios referidos a los directores escolares y a las políticas y prácticas de inclusión, de ahí la importancia, originalidad y contribución de este estudio.

La investigación realizada por Francis, Gross, Blue-Banning, Haines y Turnbull (2016) en la que se identificaron las prácticas de directivos en escuelas reconocidas como óptimas en inclusión, señala que los "directores crearon culturas escolares acogedoras e inclusivas, las que - a su vez- incentivaron la asociación y trabajo colaborativo entre padres y profesionales" (p. 54). El citado estudio realizó 11 grupos focales con padres de alumnos con y sin discapacidades en seis escuelas norteamericanas, logrando identificar las acciones realizadas por los directivos que los padres reportaban como favorecedoras de la inclusión. La Tabla 1 resume estos aspectos. 
Tabla 1

Actividades de colaboración entre directores de escuela y padres

\begin{tabular}{l}
\hline Directores de escuela \\
\hline - Crear una cultura y ambiente escolar cálido y acogedor. \\
- Pstablecer comunicación frecuente, amistosa e informal con las familias. \\
- Identificar y participar en abordar necesidadades y eventos patrocinados por la escuela. \\
- Mantener altas expectativas para el personal de la escuela. \\
- Modelar y seguir adelante con las expectativas. \\
- Distribuir el liderazgo entre el personal de la escuela y los padres.
\end{tabular}

Padres

- Participar en la toma de decisiones respecto de la educación de los alumnos.

- Proporcionar a los educadores información y estrategias acerca de los alumnos.

- Aportar al director con nuevos ideales o iniciativas.

- Iniciar, participar y/o liderar consejos escolares.

- Contribuir con tiempo, conocimientos y/o habilidades a las actividades y eventos patrocinados por la escuela.

- Cultivar la participación de los padres entre pares y representar los intereses de los padres a través de roles de liderazgo.

Fuente: Francis et al., 2016, p. 54.

Al revisar los hallazgos de este estudio es posible reconocer que estos son muy similares a los de Friend y Cook (2007), quienes identificaron seis características que determinan el éxito de la colaboración maestro-padre. Estas son: comprender que la colaboración debe ser voluntaria; la necesidad de compartir recursos; ser responsables en la toma de decisiones y apuntar a objetivos comunes; reconocer el rol de cada uno; la capacidad de trabajar y planificar juntos; y confiar y respetarse mutuamente. Estas consideraciones son importantes, ya que la relación profesorpadre/madre requiere, como mínimo, de una validación mutua de las opiniones y de la oportunidad de conversaciones francas y respetuosas, centradas en el aprendizaje del alumno, aspectos que coinciden con los resultados del estudio de Rueda, Giné y Sarrionandia (2016).

Por su parte, las conversaciones francas y colaborativas entre profesores y padres enfrentan diversas barreras. En los casos de estudiantes con discapacidad, el principal obstáculo son las percepciones negativas de los profesores y profesionales de escuela respecto de los padres de los alumnos (Blue-Banning, Summers, Nelson \& Frankland, 2004; Cooper, Riehl \& Hasan, 2010; Cullen, 
Gregory \& Noto, 2010; Hill \& Taylor, 2004). Estos, a su vez, dudan del apoyo real con el que contarán sus hijos o temen que estos experimenten acoso físico o psicológico en la escuela (Tash, 2014). Al respecto, el estudio de Jaen (2014) indaga en la percepción de madres y padres de hijos con síndrome de Down y su inclusión en la escuela, para lo cual se aplicó un cuestionario a 200 informantes, concluyendo que existe una respuesta positiva de los centros educativos, pese a ser conscientes de la falta de recursos materiales y humanos para su óptimo desarrollo. Por último, otra de las barreras que afecta la relación familia-escuela es la conciliación entre la vida laboral y familiar. Ante esto, Álvarez (2016) recomienda adecuar los horarios de los centros para posibilitar la participación familiar, lo cual coincide con los resultados de Rodríguez, González y López (2016).

La inclusión también involucra preocupaciones respecto de los riesgos de exclusión por razones socioeconómicas y/o de migración. Desde la perspectiva socioeconómica, el estudio de Julio-Maturana et al. (2016) señala que para los docentes

...la familia' (en singular) sería 'la responsable' (unicausal) de que sus estudiantes estén en condición de 'prioritarios', debido a su situación de 'pobreza'; extendiendo esta visión carenciada de bienestar socioeconómico a su condición de aprendiz, atribuyéndoles a los 'prioritarios' una identidad de aprendiz carente, que los incapacitaría de aprender por el solo hecho de ser pobres (p. 89).

Ahora bien, desde una perspectiva migratoria, el estudio de Sanz, Prados, Vicente y Martínez (2016), realizado en la comunidad de Murcia, compara la percepción de la comunicación familiaescuela entre familias de inmigrantes y españolas, concluyendo que estas últimas conocen mejor el centro educativo, participan en la vida colegial, contribuyen más al desarrollo del aprendizaje de sus hijos desde el hogar y poseen relaciones más positivas con el centro que las familias de inmigrantes. Las diferencias identificadas son estadísticamente significativas, concluyendo que las familias españolas están mejor vinculadas con los centros educativos que las familias de los inmigrantes. 
En el contexto nacional, el incremento de la migración en Chile ha implicado que en las aulas escolares de escuelas urbanas de bajos recursos económicos aumente paulatinamente el número de estudiantes de otras nacionalidades. Las investigaciones de Pavez Soto (2012) y Tijoux (2013) analizan la inserción de alumnos peruanos y ambos concluyen que la nacionalidad es utilizada como un estigma negativo, particularmente en los sectores socioeconómicos más pobres, es decir, que la socialización de los niños en barrios de inmigrantes de escasos recursos es una barrera para su desarrollo académico. Desde el punto de vista curricular, el estudio de Hernández (2016) concluye que

...las escuelas en contextos de alumnado migrante no han logrado implementar una política inclusiva que facilite la integración de los niños y niñas extranjeros. Se evidencia la ausencia de un plan y actividades concretas para eliminar la discriminación, el racismo y la xenofobia (p. 167).

Complementariamente, el estudio de Saavedra, Henríquez, Yomary, Pérez y Carrasco (2016) — que profundiza en la vivencia escolar de niños inmigrantes en la ciudad de Talca-, concluye que estos sufren situaciones de ansiedad ante lo desconocido, y que un obstáculo importante para el avance curricular es la carencia de algunas habilidades comunicativas y las estrategias pedagógicas de los docentes. "No obstante, a medida que establecen lazos afectivos, comparten juegos y trabajos escolares, se genera un reconocimiento mutuo, donde se aceptan y valoran aspectos propios de las diferentes culturas" (p. 908).

Como se ha analizado, la inclusión es multidimensional e involucra asegurar oportunidades de aprendizaje para todos los estudiantes, lo que demanda por parte del personal directivo de los establecimientos educacionales un conjunto de habilidades, conocimientos y destrezas para influir positivamente en la movilización de las prácticas docentes y en el desarrollo de relaciones de confianza entre padres y profesores. 


\section{Metodología}

\subsection{Participantes}

Los participantes corresponden a siete directores que fueron parte de un programa de formación nacional de directores de excelencia. Cuatro directivos son de género femenino y tres de género masculino. El promedio de edad corresponde a 53 años y todos han trabajado en sus respectivos cargos por más de 15 años. El grupo de directores participantes en el estudio representa a seis tipos de establecimientos: Liceo técnico-profesional de enseñanza media (1), Escuela especial (1), Escuela básica (2), Escuela de adultos (1), Liceo humanistacientífico (1), y Colegio particular subvencionado con sello católico (1).

\subsection{Técnicas de recopilación de información}

La escasa investigación nacional y el carácter de la pregunta de investigación requirió de un diseño de investigación que recogiera la multiplicidad de factores que cada director, individualmente, identificaba como condición necesaria para la instalación de prácticas educativas inclusivas, de modo de organizarlos luego colectivamente a través de clústeres (agrupaciones de declaraciones). Esto permitió avanzar desde las subjetividades individuales a las opiniones y necesidades de un colectivo que ejerce la misma función. Junto con el análisis factorial realizado y la identificación de dimensiones que subyacen en los datos, ello posibilitó comprender más profundamente el fenómeno estudiado.

Esta investigación implementó una metodología mixta que incluyó una técnica cuantitativa, análisis factorial y una técnica cualitativa denominada concept mapping (Johnson \& Onweugbuzie, 2004) que considera la creación de mapas conceptuales por los participantes (Dagenais et al., 2015; Dagenais, Ride \& Péladeau, 2012; Trochim \& Cabrera, 2005). La técnica de concept mapping es una herramienta efectiva para entender las relaciones entre diversos conceptos e ideas (Dagenais et al., 2012). Esta técnica de indagación permite comprender los nodos e interrelaciones entre conceptos para ser analizados con miras a descubrir patrones relacionales (Creswell, 2007). Asimismo, los mapas entregan una imagen representativa 
de una situación compleja que tensiona lo pensado y lo pragmático (Trochim \& Cabrera, 2005) y son fundamentalmente útiles cuando se busca la priorización, relación y repetición de ideas (Dagenais et al., 2015), por este motivo constituyen un elemento altamente significativo que se alinea con los objetivos investigativos del presente estudio.

\subsection{Etapas de la investigación}

Siguiendo la propuesta metodológica, la investigación se realizó en cuatro etapas:

- Tormenta de ideas (brainstorming). Se invitó a los participantes a contestar la siguiente pregunta: "¿La educación inclusiva en la escuela sería posible si...?". Cada participante ofreció un promedio de 15 declaraciones de respuesta.

- Jerarquización. Posteriormente, se asignó a cada participante la calificación de las otras declaraciones realizadas por los demás participantes con un número entre (1) y (5), siendo (5) el más importante y (1) el menos.

- Diseño. Finalizada la calificación de las declaraciones, se solicitó a los participantes que las agruparan en clústeres, dando a cada uno de ellos un nombre representativo de las declaraciones allí contenidas.

- Análisis factorial exploratorio. Se creó una base de datos con las declaraciones y sus valoraciones en el software SPSS versión 22.0, se realizó un análisis factorial exploratorio siguiendo las recomendaciones de Streiner (1994). Se identificaron aquí cinco dimensiones, las que se muestran en la sección resultados. Para crear el nombre de cada una, cada investigador analizó las declaraciones contenidas independientemente y seleccionó uno de los nombres creados por los directores que representaría sus contenidos; luego, ambos investigadores definieron el nombre del clúster por consenso y de forma colaborativa (Creswell, 2007).

\subsection{Recolección de datos}

Los datos fueron recogidos durante el programa de formación nacional de directores de excelencia. Se verificaron dos sesiones de 
trabajo con el grupo de siete directores seleccionados. En la primera de ellas cada directivo recibió 15 tarjetas donde debía escribir una declaración que respondiera a la pregunta: “¿La educación inclusiva sería posible si...?". Los participantes trabajaron individualmente produciendo sus declaraciones. Luego, se les entregaron copias de las declaraciones de los otros participantes en el estudio, completándose un total de 106 afirmaciones, las que fueron clasificadas por parte de los directivos utilizando los números del 1 al 5, donde 5 era muy importante y 1 , muy poco importante. Una vez finalizada la jerarquización de las aseveraciones, las organizaron alrededor de una característica unificadora, creando clústeres temáticos. En la segunda sesión cada uno de los participantes presentó y explicó su agrupación a los otros directores. Cada sesión duró aproximadamente 90 minutos.

\subsection{Análisis de datos}

El análisis de los datos se realizó de manera secuencial concurrente (Creswell, 2007). En primer término, los clústeres creados por los directores se analizaron buscando coincidencias temáticas entre las declaraciones incluidas. En segundo lugar, se creó una matriz de datos, en la que se integraron las 106 declaraciones con la jerarquización elaborada por los directores y se realizó un análisis factorial que identificó cinco dimensiones.

\section{Resultados}

Los directivos organizaron clústeres temáticos individualmente: dos de ellos elaboraron cinco clústeres; uno organizó tres; dos desarrollaron ocho; uno diez y otro once. En total, se crearon 50 clústeres, que se reducen a 27 por las coincidencias temáticas, como se muestra en la Tabla 2. Los clústeres que reunieron un mayor número de coincidencias fueron los de políticas públicas $(n=6)$; recursos $(n=$ 5); cultura $(n=4)$; y actitud personal, escuela, capacitación y familia $(n=3)$, como se muestra en la Tabla 2. Es importante observar que la coincidencia en los nombres de los clústeres no implica coincidencia en las declaraciones y jerarquización. 
El clúster relacionado con actitud personal $(n=59)$ fue el que reunió el mayor número de aseveraciones jerarquizadas como muy importantes, seguido del de cultura $(n=37)$. En el extremo opuesto se encuentran los clústeres de familia $(n=0)$ y de sensibilización $(n=1)$.

Tabla 2

Frecuencia de clúster

\begin{tabular}{|c|c|c|c|c|c|c|c|c|}
\hline$\ominus$ & 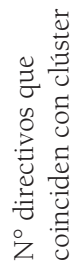 & 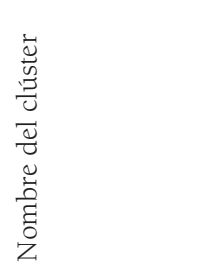 & 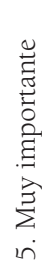 & 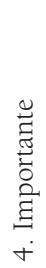 & 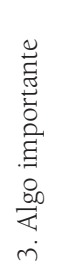 & 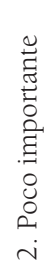 & 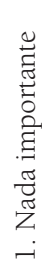 & 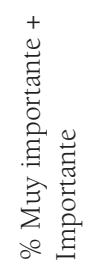 \\
\hline 1 & 4 & Cultura & 37 & 29 & 14 & 14 & 8 & $16,0 \%$ \\
\hline 2 & 3 & Actitud personal & 59 & 0 & 1 & 1 & 0 & $14,3 \%$ \\
\hline 3 & 1 & Gestión & 24 & 5 & 1 & 0 & 0 & $7,0 \%$ \\
\hline 4 & 1 & Convivencia & 22 & 6 & 4 & 0 & 0 & $6,8 \%$ \\
\hline 5 & 1 & Respeto & 19 & 9 & 1 & 0 & 0 & $6,8 \%$ \\
\hline 6 & 3 & Escuela & 12 & 15 & 30 & 22 & 6 & $6,6 \%$ \\
\hline 7 & 3 & Capacitación & 9 & 10 & 4 & 0 & 0 & $4,6 \%$ \\
\hline 8 & 1 & Barreras & 16 & 1 & 1 & 0 & 0 & $4,1 \%$ \\
\hline 9 & 2 & Aula & 8 & 9 & 2 & 0 & 0 & $4,1 \%$ \\
\hline 10 & 6 & Política & 4 & 12 & 20 & 27 & 16 & $3,9 \%$ \\
\hline 11 & 1 & Valores & 4 & 9 & 0 & 1 & 0 & $3,2 \%$ \\
\hline 12 & 2 & Profesor & 3 & 9 & 0 & 0 & 0 & $2,9 \%$ \\
\hline 13 & 1 & Cambio & 6 & 5 & 4 & 4 & 4 & $2,7 \%$ \\
\hline 14 & 5 & Recursos & 4 & 6 & 8 & 4 & 0 & $2,4 \%$ \\
\hline 15 & 1 & Formación & 7 & 2 & 1 & 0 & 0 & $2,2 \%$ \\
\hline 16 & 1 & Excusas & 4 & 4 & 14 & 5 & 1 & $1,9 \%$ \\
\hline 17 & 1 & Sistema educativo & 4 & 4 & 1 & 2 & 0 & $1,9 \%$ \\
\hline 18 & 2 & Infraestructura & 4 & 3 & 1 & 2 & 0 & $1,7 \%$ \\
\hline 19 & 1 & Estados de ánimo & 4 & 2 & 0 & 0 & 0 & $1,5 \%$ \\
\hline 20 & 1 & Directivo & 1 & 5 & 0 & 1 & 1 & $1,5 \%$ \\
\hline 21 & 1 & Emociones & 3 & 1 & 0 & 0 & 0 & $1,0 \%$ \\
\hline 22 & 1 & NNE & 0 & 4 & 0 & 2 & 0 & $1,0 \%$ \\
\hline 23 & 1 & Resolución & 3 & 0 & 0 & 0 & 0 & $0,7 \%$ \\
\hline 24 & 1 & Tiempo & 1 & 1 & 0 & 0 & 0 & $0,5 \%$ \\
\hline 25 & 1 & Multidisciplinario & 0 & 2 & 2 & 1 & 0 & $0,5 \%$ \\
\hline 26 & 1 & Sensibilización & 0 & 1 & 2 & 0 & 0 & $0,2 \%$ \\
\hline 27 & 3 & Familia & 0 & 0 & 1 & 2 & 0 & $0,0 \%$ \\
\hline \multicolumn{3}{|c|}{$\begin{array}{l}\text { Total } \\
\text { porcentual }\end{array}$} & & & & & & $100 \%$ \\
\hline
\end{tabular}

Fuente: Elaboración propia. 
Un $36,7 \%$ de las declaraciones fueron jerarquizadas en nivel 5 , es decir, como muy importante; el 24,9\% en un nivel 4, esto es, importante; el 19,1\% en un nivel 3, o sea, algo importante; el $12,9 \%$ calificó con un nivel 2, poco importante; y el 5,7\% en un nivel 1, esto es, nada importante. Esto significa que el 61,6\% de las declaraciones son valoradas como importantes o muy importantes por los participantes.

De las 106 declaraciones, solo cuatro fueron valoradas como muy importantes por todos los participantes: (Una educación inclusiva sería posible en la escuela si...) existe respeto y tolerancia; existe más respeto; aceptamos la diversidad; y somos capaces de mirar y tratar a los demás con respeto.

Como se puede observar, las cuatro declaraciones aluden a aspectos actitudinales, asociados al respeto y a la aceptación.

El análisis factorial identificó cinco dimensiones, donde cada una incluye declaraciones diferentes que fueron categorizadas por parte del equipo de investigación utilizando los nombres de los clústeres creados por los propios participantes. La Tabla 3 muestra las aseveraciones de la primera desviación estándar, que como se observa está compuesta por aseveraciones de diferentes clústeres. 
Tabla 3

Análisis factorial de clúster

\begin{tabular}{|c|c|c|}
\hline Nombre clúster & Declaraciones & Desviación estándar \\
\hline Políticas públicas & $\begin{array}{l}\text { Si existieran más leyes que protejan la } \\
\text { inclusión. }\end{array}$ & 0,926 \\
\hline Recursos humanos & $\begin{array}{l}\text { Si cada escuela contara con los especialistas } \\
\text { que se requieren. }\end{array}$ & 0,891 \\
\hline Recursos humanos & $\begin{array}{l}\text { Si tuviéramos especialistas disponibles para } \\
\text { apoyar la labor docente. }\end{array}$ & 0,878 \\
\hline Recursos materiales & $\begin{array}{l}\text { Si dispusiéramos de material didáctico para } \\
\text { ello. }\end{array}$ & 0,861 \\
\hline Recursos materiales & $\begin{array}{l}\text { Si los recursos didácticos fueran mejor } \\
\text { utilizados. }\end{array}$ & 0,85 \\
\hline Recursos materiales & Si aumentaran los recursos. & 0,841 \\
\hline Recursos financieros & $\begin{array}{l}\text { Si existieran las condiciones económicas } \\
\text { para hacerlo. }\end{array}$ & 0,837 \\
\hline Políticas públicas & $\begin{array}{l}\text { Si la sociedad chilena estuviera sensibilizada } \\
\text { y entendiera las discapacidades. }\end{array}$ & 0,828 \\
\hline Recursos financieros & $\begin{array}{l}\text { Si tuviéramos recursos para atender las } \\
\text { individualidades. }\end{array}$ & 0,827 \\
\hline Recursos humanos & $\begin{array}{l}\text { Si los sostenedores pudieran mejorar las } \\
\text { condiciones de contrato de los especialistas. }\end{array}$ & 0,819 \\
\hline Políticas públicas & $\begin{array}{l}\text { Si las políticas públicas estuvieran bien } \\
\text { explicadas y adaptadas a las diferentes } \\
\text { realidades, incluyendo toda la diversidad. }\end{array}$ & 0,803 \\
\hline Recursos humanos & $\begin{array}{l}\text { Si existieran equipos multidisciplinarios para } \\
\text { apoyar la tarea educativa. }\end{array}$ & 0,775 \\
\hline Políticas públicas & $\begin{array}{l}\text { Si tuviéramos todos conocimiento de la Ley } \\
\text { de Inclusión y sus decretos }\end{array}$ & 0,766 \\
\hline Formación & Si se preparara mejor en convivencia. & 0,707 \\
\hline Recursos humanos & Si existiera capacitación específica. & 0,707 \\
\hline Aula & $\begin{array}{l}\text { Si se organizaran las actividades de manera } \\
\text { natural, igual y equitativa para todos los } \\
\text { alumnos. }\end{array}$ & 0,705 \\
\hline Actitud personal & Si se sensibilizara a mi comunidad. & 0,705 \\
\hline Recursos materiales & $\begin{array}{l}\text { Si todas las escuelas contaran con la } \\
\text { infraestructura correspondiente. }\end{array}$ & 0,704 \\
\hline Recursos materiales & Si existieran clases con menos estudiantes. & 0,683 \\
\hline Recursos materiales & $\begin{array}{l}\text { Si las salas de clases fueran espacios } \\
\text { propicios para implementar diseños } \\
\text { universales de aprendizaje (DUA). }\end{array}$ & 0,672 \\
\hline Formación & $\begin{array}{l}\text { Si la formación inicial de los profesores } \\
\text { incluyera un módulo con el tema. }\end{array}$ & 0,665 \\
\hline Actitud personal & $\begin{array}{l}\text { Si todos ayudáramos a entender al otro } \\
\text { (discapacidades, diferencias étnicas). }\end{array}$ & 0,661 \\
\hline Actitud personal & Si supiéramos qué es inclusión. & 0,649 \\
\hline Recursos financieros & $\begin{array}{l}\text { Si tuviéramos acceso al financiamiento para } \\
\text { este fin. }\end{array}$ & 0,649 \\
\hline Formación & $\begin{array}{l}\text { Si se preparara adecuadamente a todos los } \\
\text { que trabajan en educación. }\end{array}$ & 0,639 \\
\hline
\end{tabular}

Fuente: Elaboración propia. 
Con la finalidad de profundizar en el análisis, se desarrolló la Tabla 4, en la que para cada desviación estándar se asociaron las declaraciones a una de las dimensiones. En ella se evidencia que la primera desviación estándar agrupa las aseveraciones relativas a recursos materiales y humanos para llevar a cabo la inclusión. La segunda desviación reúne las aseveraciones asociadas a la actitud personal y políticas públicas. En la tercera, cuarta y quinta desviación estándar no se observan agrupaciones de declaraciones relevantes, sino que se trata de aseveraciones amplias (por ejemplo: si todos fuéramos buenos).

Tabla 4

Organización interna de cada clúster

\begin{tabular}{lccccc}
\hline Declaración & $\begin{array}{c}\text { Primera } \\
\text { desviación }\end{array}$ & $\begin{array}{c}\text { Segunda } \\
\text { desviación }\end{array}$ & $\begin{array}{c}\text { Tercera } \\
\text { desviación }\end{array}$ & $\begin{array}{c}\text { Cuarta } \\
\text { desviación }\end{array}$ & $\begin{array}{c}\text { Quinta } \\
\text { desviación }\end{array}$ \\
\hline Capacitaciones & 3 & 4 & 2 & 0 & 0 \\
\hline Sala de clases & 1 & 0 & 0 & 0 & 0 \\
\hline Políticas públicas & 4 & 14 & 0 & 0 & 1 \\
\hline Recursos & 33 & 2 & 0 & 1 & 1 \\
\hline Misceláneo & 3 & 0 & 0 & 0 & 0 \\
\hline Actitud personal & 4 & 20 & 0 & 6 & 4 \\
\hline Directores & 0 & 2 & 2 & 0 & 0 \\
\hline Estrategias de & 0 & 2 & 1 & 1 & 3 \\
enseñanza & 0 & 0 & 2 & 0 & 0 \\
\hline Profesores & & & & & 0
\end{tabular}

Fuente: Elaboración propia.

La Figura 1 ratifica lo expresado más arriba en relación con el predominio de la primera desviación estándar sobre las siguientes. 


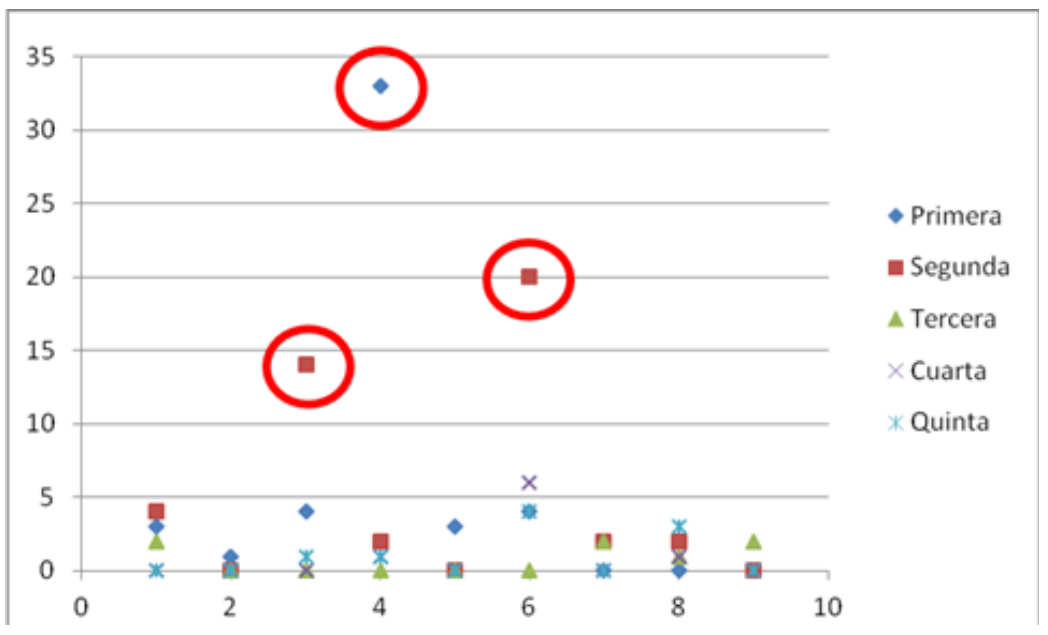

Figura 1. Distribución del clúster.

Fuente: Elaboración propia.

\section{Discusión}

Del análisis de datos se evidencia que los directivos, al responder a la pregunta "¿La educación inclusiva en la escuela sería posible si...?”, concentran sus preocupaciones en tres grandes áreas: recursos (financieros, materiales y humanos), actitudes personales (que incluye aseveraciones asociadas con aspectos culturales) y políticas públicas de inclusión. Estos tres aspectos se encuentran íntimamente relacionados.

\subsection{Recursos humanos y económicos}

Se trata de aspectos clave para gestionar y liderar las organizaciones escolares (Anderson, 2008; Bellei et al., 2004; Hargreaves \& Shirley, 2012; Harris, 2009) y se transforman en una oportunidad en la medida en que los directores cuentan con ellos para poder gestionarlos. En el caso chileno, usualmente la autonomía para poder decidir qué hacer con los recursos es reducida (Weinstein \& Muñoz, 2014). No obstante, los recursos pueden servir como una alternativa interesante para generar las condiciones necesarias para emplazar prácticas de inclusión. Esto lo indican los datos, siendo la primera desviación estándar que afecta en la generación de condiciones 
inclusivas dentro de las escuelas, lo que se encuentra reflejado en declaraciones asociadas con disponer de recursos humanos especializados y recursos didácticos adecuados. Por eso es necesario pensar en qué medida los directores pueden decidir internamente qué y cómo distribuir los recursos para poder instalar prácticas inclusivas dentro de sus respectivas escuelas.

\subsection{Actitudes personales}

La actitud de las personas que componen las organizaciones influye en el ambiente (López et al., 2014). En el caso de las prácticas de inclusión, la actitud personal constituye un elemento de exclusión para los directores. Es decir, se necesita cambiar la actitud de las personas para forjar entornos propicios para instalar prácticas inclusivas. En palabras de los participantes de este estudio ello implica desarrollar la sensibilidad de los profesores y de la comunidad entregando información, promoviendo el compromiso y el desarrollo de prácticas inclusivas. Desde la mirada de Infante (2010), esto puede ser explicado porque la inclusión no regula solamente las prácticas pedagógicas dentro del aula, sino también y, primordialmente, las ideas en torno al significado de la otredad. Esto hace referencia a situar al otro desde un espacio de exclusión que tiende a ser reproducido por los sujetos cohabitantes dentro del contexto sociocultural de las escuelas (Echeita y Ainscow, 2011; Infante, 2010). En este sentido, la actitud personal en la escuela debe ser un elemento a considerar para instalar prácticas inclusivas.

El desafío para los directores escolares está puesto en cómo poder influir y gestionar la actitud de aquellos que presentan resistencia a la inclusión, simbolizándola como una carga adicional al trabajo pedagógico. Tradicionalmente las aulas inclusivas representan un desafío para los profesores y directores, y pueden ser consideradas como un obstáculo para el aprendizaje (Cuadra, 2008; Fernández, 2012). Ello, porque atentan contra principios reguladores y de exclusión ampliamente adoptados por el sistema educacional chileno (Drago \& Paredes, 2011; García-Huidobro, 2007; González, 2003; Levin, 2011). Así, para los profesores y directivos el tránsito desde políticas de integración hacia políticas de inclusión es un proceso 
que demanda tiempo, convicción y participación para desarrollar una cultura escolar basada en valores inclusivos (Villegas, Simón y Echeita, 2014), así como también se trata de un proceso continuo (Kluth, Biklen, English-Sand \& Smukler, 2007).

\subsection{Políticas públicas}

Establecen un marco referencial sobre el actuar y proceder de las escuelas, y específicamente respecto de las funciones, tareas y responsabilidades de los directores escolares. A partir de los datos, los directores de este estudio señalan que las políticas públicas deben ser de apoyo a la inclusión, creando lineamientos generales para desarrollar prácticas inclusivas dentro de las escuelas. Sin embargo, las políticas públicas de inclusión en Chile se han caracterizado por estar sujetas a un sistema de rendición de cuentas y no necesariamente se transforman en una contribución a la labor directiva (Luna, 2015). Este sistema ha influido negativamente en los procesos de instalación de prácticas principalmente en dos aspectos clave: la labor docente y su profesionalización, y la falta de motivación en la enseñanza (Luna, 2015). La idea es apostar por políticas públicas de apoyo a la inclusión que no se manifiesten como constructos aislados, desconectados y ajenos a la realidad percibida dentro de las escuelas. Esto, porque para los directores es fundamental poseer claridad acerca de los lineamientos de la política pública, ya que el rol directivo se encuentra sustentado en la capacidad de orientación, guía e influencia en su equipo, y para ello es necesario poseer un norte claro emanado desde estas: al parecer ello no estaría del todo resuelto en el caso chileno. Así, se abre una oportunidad de exploración investigativa, pensando en cómo las políticas públicas permiten o no orientar el trabajo de los directores escolares en la instalación, monitoreo y evaluación de prácticas inclusivas.

Las tres condiciones expuestas son variables externas que indudablemente influyen en el desarrollo de prácticas inclusivas en la escuela y que vuelven a plantear un problema de congruencia y superposición macroestructural inherente a la realidad chilena. Este problema se suscita a causa de la incapacidad de construir un encuentro entre el mundo de la política pública y el escolar. Ambos 
mundos, situados en esferas distantes, suelen toparse solo cuando uno de ellos busca imponer algo sobre el otro. La reflexión clave es cómo hacer dialogar a ambos, sin que por ello se deban superponer y/o suprimir ideas. Pareciera ser que el liderazgo e inclusión pueden contribuir a resolver estos desencuentros.

Así, puesto que el diálogo entre las políticas públicas y la escuela es realizado por el director, este necesita ser líder pedagógico para mejorar y cambiar las escuelas, siendo su principal tarea motivar, capacitar y comprometer a los profesores para alcanzar mejores resultados en sus estudiantes (Leithwood et al., 2008). Pese a esta premisa, en las declaraciones presentadas no existió ninguna variable asociada con acciones que tendieran al aprendizaje de los estudiantes o que pudieran estar vinculadas con el liderazgo pedagógico. Esto coincide con hallazgos de estudios recientes (Quiroga y Aravena, 2018) en donde los directores demuestran poseer escasos datos respecto de los niveles de aprendizaje de los estudiantes, lo que impide el impacto más directo en el cambio de prácticas docentes y especialmente en el núcleo pedagógico. De ahí que resulte clave promover un liderazgo pedagógico que lleve a los directores a concentrar sus esfuerzos en la mejora escolar, específicamente ocupándose de lo que sucede en el aula.

Para graficar este aspecto, de las 106 declaraciones, solo cuatro de ellas se referían a los propios directores y su quehacer: (Una educación inclusiva sería posible en la escuela si...) se considera en el PEI (Proyecto Educativo Institucional); los directores tuvieran más instrumentos para administrar los recursos; los directores tuvieran más autonomía en la gestión escolar; y los directores fueran inclusivos.

Como se aprecia, estas declaraciones están relacionadas con aspectos de la gestión administrativa, pero no con el aprendizaje de los estudiantes o el desarrollo de nuevas habilidades y capacidades docentes para afrontar entornos diversos. Con ello, es posible advertir que los directores condicionan la inclusión en sus establecimientos a circunstancias externas, en lugar de vincularla con acciones internas que podrían depender exclusivamente de su capacidad de influencia y desempeño profesional. 


\section{Conclusiones}

Las prácticas inclusivas en los establecimientos escolares chilenos son de reciente abordaje. Por lo que los resultados de este estudio aportan identificando las condiciones que los directivos consideran importantes para la implementación de estas prácticas: recursos con autonomía en su asignación, políticas públicas claras y desarrollo de actitudes personales positivas hacia grupos culturales, económicos y sociales diferentes, así como también aquellas situaciones asociadas a la formación y estrategias docentes con foco inclusivo.

Junto con el fortalecimiento de las condiciones externas es importante asegurar también las condiciones internas de las organizaciones escolares para la inclusión, que según Booth y Ainscow (2015) se deben concebir desde una perspectiva sistémica. En este sentido, todos los profesores deberían responder con equidad a la diversidad del alumnado, no solo los especialistas, por lo que la inclusión se extiende a los profesionales de la escuela y a las familias de los estudiantes (McKinney, Labat \& Labat, 2015). Esto puede ser profundizado a través de las conclusiones de un reciente estudio realizado en Malasia, que establece que las aulas inclusivas exitosas incluyen la interacción colaborativa entre los maestros y los padres (Adams, Harris \& Jones, 2016) ratificado en el estudio de Lyons, Thompson y Timmons (2016). El supuesto que subyace es que todos los interesados en el bienestar y aprendizaje de los estudiantes pueden participar creativamente en su aprendizaje, ya que la desconexión entre ambos actores afecta su experiencia de aprendizaje (Staples $\&$ Diliberto, 2010).

De estas conclusiones de investigaciones internacionales se desprende la importancia de la relación profesores-padres/madres. En este estudio un solo directivo elaboró el clúster "familia", el que quedó constituido por tres declaraciones, una jerarquizada con valor 3 (medianamente importante) y dos jerarquizadas con valor 2 (poco importante). Esto implica que la familia es poco importante al momento de identificar y valorar las condiciones necesarias para lograr la inclusión en su escuela. Esta invisibilización de la relación familia-escuela es un aspecto que inhibe la eliminación de 
barreras socioculturales entre docentes y padres/madres de familia, estimulando explicaciones culpabilizadoras y de desconfianza.

De los resultados del estudio, se recomienda orientar los procesos formativos de directivos docentes en la instalación de prácticas inclusivas. En primer término, es necesario que los directivos comprendan que las relaciones de confianza y colaboración con los padres/madres es primordial para la instalación de prácticas inclusivas. El eje de este esfuerzo debe estar en el bienestar y en el aprendizaje de los estudiantes y no en proyectar prejuicios socioeconómicos y culturales, especialmente, con estudiantes en condiciones de pobreza, inmigrantes o con dificultades cognitivas. Como afirman las investigaciones de Epstein, Galindo y Sheldon (2011) y Gurr, Drysdale y Mulford (2005), los directores que facilitan y aprovechan el capital social, colaborando con los padres, mejoran los resultados de los involucrados.

En segundo término, se debe contemplar el desarrollo de prácticas colaborativas de planificación y análisis de resultados de los estudiantes en el desarrollo profesional de los directores, con la finalidad de potenciar la creatividad y diseño de respuestas pertinentes a las necesidades de los estudiantes. Finalmente, es fundamental el desarrollo de una responsabilidad individual y colectiva sobre los aprendizajes de los estudiantes que sea abordada por los programas de desarrollo profesional para directivos y que, combinada con una percepción de eficacia individual y colectiva, permita sostener altas expectativas sobre su propio trabajo y los aprendizajes de los estudiantes.

En cuanto a los límites y proyecciones de esta investigación se puede indicar que los directivos escolares, como líderes pedagógicos en sus establecimientos son clave para implementar las políticas públicas y las innovaciones. Por lo mismo, es importante indagar acerca de cuáles serían las condiciones necesarias para la instalación de prácticas educativas inclusivas. Las conclusiones de este estudio representan una base inicial para sucesivas investigaciones que puedan replicar la metodología con poblaciones más amplias y/o focalizarse en instituciones adscritas al nuevo sistema de admisión 
escolar, donde probablemente aumentará la heterogeneidad de los estudiantes y las escuelas serán desafiadas a implementar prácticas de inclusión.

Aunque la muestra de este estudio no es representativa, la evidencia que proporciona sí es indicativa y sirve como punto de referencia para formadores de directivos y encargados de formular políticas públicas, así como también para identificar las preocupaciones de los directivos docentes y anticipar las necesidades que pueden presentarse en el corto plazo, además de contribuir a reducir la brecha de evidencia empírica en la literatura acerca de la relación entre liderazgo e inclusión en Chile.

\section{Referencias}

Adams, D., Harris, A., \& Jones, M. S. (2016). Teacher-parent collaboration for an inclusive classroom: Success for every child. Malaysian Online Journal of Educational Sciences, 4(3), 58-71. Recuperado de http:// eprints.um.edu.my/17118/

Álvarez, L. F. F. (2016). Análisis de la visión del profesorado sobre la dificultad de las madres para participar en el centro escolar de educación infantil y primaria. En J. J. Gázquez, M. M. Molero, M. C. Pérez-Fuentes, M. M. Simón, A. B. Barragán y Á. Martos Martínez (Coords.), Investigación en el ámbito escolar: un acercamiento multidimensional a las variables psicológicas y educativas. Volumen II (127-134). Recuperado de https://dialnet.unirioja.es/descarga/libro/682131.pdf

Anderson, J. (2008). Principals' role and public primary schools' effectiveness in four Latin American cities. The Elementary School Journal, 109(1), 36-60. https://doi.org/10.1086/592366

Aravena, F. \& Quiroga, M. (2016). Neoliberalism and education on an international perspective: Chile as perfect scenario. Bulgarian Journal of Science E Education Policy, 10(1), 1-21. Recuperado de http://oaji. net/articles/2016/585-1470755962.pdf

Bellei, C., Muñoz, G., Pérez, L., y Raczynski, D. (2004). ¿Quién dijo que no se puede? Escuelas efectivas en sectores de pobreza. Santiago de Chile: Unicef-Mineduc.

Blue-Banning, M., Summers, J. A., Nelson, L. L., \& Frankland, C. (2004). Dimensions of family and professional partnerships: Constructive guidelines for collaboration. Exceptional Children, 70(2), 167-184. https://doi.org/10.1177/001440290407000203 
Booth, T. y Ainscow. M. (2015). Guía para la educación inclusiva. Desarrollando el aprendizaje y la participación en los centros escolares. Madrid: OEI.

Cavieres, E. (2011). The class and culture-based exclusion of the Chilean neoliberal educational reform. Educational Studies: A Journal of the American Educational Studies Association, 47(2), 111-132. https://doi.org/10.1080/00131946.2011.554588

Creswell, W. (2007). Qualitative inquiry \& research design: Choosing among five approaches (2nd ed.). Thousand Oaks, CA: SAGE.

Cooper, C. W., Riehl, C. J., \& Hasan, A. L. (2010). Leading and learning with diverse families in schools: Critical epistemology amid communities of practice. Journal of School Leadership, 20(6), 758-788.

Cuadra, D. J. (2008). Representaciones sociales de niño integrado y proyecto de integración escolar en escuelas básicas con y sin integración. Revista Electrónica Iberoamericana sobre Calidad, Eficacia y Cambio en Educación (REICE), 6(3), 121-138. Recuperado de http://www.rinace.net/arts/ vol6num3/art5.pdf

Cullen, J. P., Gregory, J. L., \& Noto, L. A. (2010). The teacher attitudes toward inclusion scale (TATIS) technical report. Carrollton, GA: Eastern Educational Research Association.

Dagenais, C., Pinard, R., St-Pierre, M., Briand-Lamarche, M., Cantave, A. K., \& Péladeau, N. (2015). Using concept mapping to identify conditions that foster knowledge translation from the perspective of school practitioners. Research Evaluation, 25(1), 70-78.

https://doi.org/10.1093/reseval/rvv026

Dagenais, C., Ridde, V., \& Peladeau, N. (octubre, 2012). The concept mapping methodology: A review of users. Trabajo presentado en 26th Annual Conference of the American Evaluation Association, Minneapolis, Minnesota.

Darling-Hammond, L., Meyerson, D., La Pointe, M., \& Orr, M. T. (2010). Preparing principals for a changing world: Lessons from effective school leadership programs. San Francisco: Jossey-Bass.

Donoso, S., Benavides, N., Cancino, V., Castro, M., y López, L. (2011). Análisis crítico de las políticas de formación de directores escolares en Chile: 1980-2010. Revista Brasileira de Educação, 17(49), 133-158. https://doi.org/10.1590/s1413-24782012000100008

Drago, J. \& Paredes, R. (2011). The quality gap in Chile's education system. CEPAL Review, 104, 16-174. Recuperado de https://www.cepal.org/ publicaciones/xml/8/45338/rvil04dragoparedes.pdf 
Echeita, G. y Ainscow, M. (2011). La educación inclusiva como derecho: marco de referencia y pautas de acción para el desarrollo de una revolución pendiente. Revista de Didáctica de la Lengua y la Literatura, 12, 26-46. Recuperado de https://dialnet.unirioja.es/descarga/ articulo/3736956.pdf

Epstein, J., Galindo, C., \& Sheldon, S. (2011). Levels of leadership: Effects of district and school leaders on the quality of school programs of family and community involvement. Education Administration Quarterly, 47(3), 462-495. https://doi.org/10.1177/0013161x10396929

Fernández, J. (noviembre, 2012). Dirección y buenas prácticas educativas en centros de orientación educativa. Trabajo presentado en el I Congreso Virtual Internacional sobre Innovación Pedagógica y Praxis Educativa (INNOVAGOGIA). Recuperado de https://www.upo.es/ocs/index.php/ innovagogia2012/Iinnovagogia2012/schedConf/presentations

Francis, G. L., Gross, J. M., Banning, M. B., Haines, S., y Turnbull, A. P. (2016). Directores escolares y padres que logran resultados óptimos: lecciones aprendidas de seis escuelas norteamericanas que han implementado prácticas inclusivas. Revista Latinoamericana de Educación Inclusiva, 10(1), 43-60. https://doi.org/10.4067/s0718-73782016000100004

Friend, M. \& Cook, L. (2007). Interactions: Collaboration skills for school professionals. Boston, MA: Allyn and Bacon.

García-Huidobro, J. E. (2007). Desigualdad educativa y segmentación del sistema escolar. Consideraciones a partir del caso chileno. Pensamiento Educativo, 40(1), 65-86. Recuperado de http://pensamientoeducativo. uc.cl/index.php/pel/article/download/399/817

Gauri, V. (1998). School choice in Chile: Two decades of educational reforms. USA: Pittsburgh Press.

González, P. (2003). Estructura institucional, recursos y gestión en el sistema escolar chileno. En C. Cox (Ed.), Políticas educacionales en el cambio de siglo. La reforma del sistema escolar en Chile (pp. 597-660). Santiago de Chile: Editorial Universitaria.

Gurr, D., Drysdale, L., \& Mulford, B. (2005). Successful principal leadership: Australian case studies. Journal of Educational Administration, 43(6), 539-551. https://doi.org/10.1108/09578230510625647

Hargreaves, A. \& Shirley, D. (2012). The global fourth way. Thousand Oaks, CA: Corwin.

Harris, A. (2009). Distributed leadership, different perspectives. London: Springer. 
Hernández, A. (2016). El currículo en contextos de estudiantes migrantes: las complejidades del desarrollo curricular desde la perspectiva de los docentes de aula. Estudios Pedagógicos (Valdivia), 42(2), 151-169. https://doi.org/10.4067/s0718-07052016000200009

Hill, N. E. \& Taylor, L. C. (2004). Parental school involvement and children's academic achievement: Pragmatics and issues. Current Directions in Psychological Science, 13(4), 161-164.

https://doi.org/10.1111/j.0963-7214.2004.00298.x

Horn, A. y Marfán, J. (2010). Relación entre liderazgo educativo y desempeño escolar: revisión de la investigación en Chile. Psicoperspectivas, 9(2), 82-104. https://doi.org/10.5027/psicoperspectivas-vol9-issue2fulltext-116

Hsieh, C. \& Urquiola, M. (2006). The effects of generalized school choice on achievement and stratification: Evidence from Chile's voucher program. Journal of Public Economics, 90(8-9), 1477-1503.

https://doi.org/10.1016/j.jpubeco.2005.11.002

Infante, M. (2010). Desafíos a la formación docente: inclusión educativa. Estudios Pedagógicos (Valdivia), 36(1), 287-297. https://doi.org/10.4067/s0718-07052010000100016

Jaén, A. M. B. (2014). La inclusión educativa desde la voz de los padres. Revista de Educación Inclusiva, 7(1), 110-120. https://dialnet.unirioja. es/servlet/articulo? codigo $=4772626$

Johnson, B. \& Onwuegbuzie, A. (2004). Mixed methods research: A research paradigm whose time has come. Educational Researcher, 33(7), 14-26. Recuperado de http://edr.sagepub.com/cgi/content/abstract/33/7/14

Julio-Maturana, C., Conejeros-Solar, L., Rojas Aravena, C., Mohammad Jiménez, M., Rubí Castillo, Y., y Cortés León, Y. (2016). Desencuentro cultural en el aula: una barrera al aprendizaje de niños y niñas en situación de pobreza. Revista Mexicana de Investigación Educativa, 21(68), 71-94. Recuperado de https://dialnet.unirioja.es/descarga/ articulo/5429562.pdf

Kluth, P., Biklen, D., English-Sand, P., \& Smukler, D. (2007). Going away to school. Stories of families who move to seek inclusive educational experiences for their children with disabilities. Journal of Disability Policy Studies, 18(1), 43-56. https://doi.org/10.1177/10442073070180010501

Leithwood, K., Harris, A., \& Hopkins, D. (2008). Seven strong claims about successful school leadership. School Leadership and Management, 28(1), 27-42. https://doi.org/10.1080/13632430701800060 
Levin, B. (2011). Chile, Latin America, and inequality in education. Phi Delta Kappan international, 93(2), 74-75.

https://doi.org/10.1177/003172171109300218

Ley $n^{\circ} 20.845$ de Inclusión escolar que regula la admisión de los y las estudiantes, elimina el financiamiento compartido y prohíbe el lucro en establecimientos educacionales que reciben aportes del Estado, del Ministerio de Educación, artículo 1, letra K. Diario Oficial de la República de Chile, Santiago, Chile, 8 de junio de 2015.

López, V., Julio, C., Morales, M., Rojas, C., y Pérez, M. V. (2014). Barreras culturales para la inclusión: políticas y prácticas de integración en Chile. Santiago de Chile: Ministerio de Educación.

Luna, L. (2015). Construyendo "la identidad del excluido": etnografía del aprendizaje situado de los niños en una escuela básica municipal de Chile. Estudios Pedagógicos (Valdivia), 41(especial), 97-114. https://doi.org/10.4067/s0718-07052015000300007

Lyons, W. E., Thompson, S. A., \& Timmons, V. (2016). 'We are inclusive. We are a team. Let's just do it': Commitment, collective efficacy, and agency in four inclusive schools. International Journal of Inclusive Education, 20(8), 889-907. https://doi.org/10.1080/13603116.2015.1122841

McKinney, C. L., Labat, M. B., \& Labat, C. A. (2015). Traits possessed by principals who transform school culture in national blue-ribbon schools. Academy of Educational Leadership, 19(1), 152-166. Recuperado de https://www.abacademies.org/articles/aeljvol19no12015.pdf

Mizala, A. \& Romaguera, P. (1998). School performance and choice: The Chilean experience. The Journal of Human Resources, 35(2), 394-417. https://doi.org/10.2307/146331

Mizala, A. \& Torche, F. (2012). Bringing the schools back in: The stratification of educational achievement in the Chilean voucher system. International Journal of Educational Development, 32(1), 132-144. https://doi.org/10.1016/j.ijedudev.2010.09.004

Pavez Soto, I. (2012). Immigration and racism: Experiences of Peruvian childhood in Santiago, Chile. Si Somos Americanos, 12(1), 75-99. https://doi.org/10.4067/s0719-09482012000100004

Quiroga, M. y Aravena, F. (2018). ¿Qué tipo de datos recolectan los directores? Implicancias para la elaboración de planes de mejora. Páginas de Educación, 11(2), 24-39. https://doi.org/10.22235/pe.v11i2.1639

Rodríguez, B., González, R. A. M., y López, M. J. R. (2016). Dificultades de las familias para participar en los centros escolares. Revista Latinoamericana de Educación Inclusiva, 10(1), 79-98.

https://doi.org/10.4067/s0718-73782016000100005 
Simón, C. S., Giné, C. G., y Echeita, G. E. (2016). Escuela, familia y comunidad: construyendo alianzas para promover la inclusión. Revista Latinoamericana de Educación Inclusiva, 10(1), 25-42.

Sapelli, C. (2003). The Chilean voucher system: Some new results and research challenges. Cuadernos de Economía, 40(121), 530-538. https://doi.org/10.4067/s0717-68212003012100020

Sanz, M. P. G., Prados, M. Á. H., Vicente, M. Á. G., y Martínez, J. P. (2016). Familia, inmigración y comunicación con el centro escolar: un estudio comparativo. Educación XX1, 19(2).

https://doi.org/10.5944/educxx1.16457

Saavedra, R. A., Henríquez, S. S., Yomary, L. G., Pérez, E. M., y Carrasco, C. N. (2016). La escuela como espacio privilegiado de integración de los niños inmigrantes. Investigación Cualitativa en Educación, CIAIQ2016, 1, 900-909. Recuperado de https://proceedings.ciaiq.org/index.php/ ciaiq2016/article/view/683/671

IBM Corp. Released 2013. IBM SPSS Statistics for Windows, Version 22.0. Armonk, NY: IBM Corp.

Streiner, D. L. (1994). Figuring out factors: The use and misuse of factor analysis. The Canadian Journal of Psychiatry, 39(3), 135-140. https://doi.org/10.1177/070674379403900303

Staples, K. E. \& Diliberto, J. A. (2010). Guidelines for successful parent involvement working with parents of students with disabilities. Teaching Exceptional Children, 42(6), 58-63. https://doi.org/10.1177/004005991004200607

Tash (2014). Dispelling the myths of inclusion education. Recuperado de http:// tash.org/wp-content/uploads/2015/04/Myths-of-IE.pdf

Tijoux, M. E. (2013). Las escuelas de la inmigración en la ciudad de Santiago: elementos para una educación contra el racismo. Polis (Santiago), 12(35), 287-307. https://doi.org/10.4067/s0718-65682013000200013

Trochim, W. M. \& Cabrera, D. A. (2005). The complexity of concept mapping. ECO, 7(1), 11-22. Recuperado de https://pdfs.semanticscholar. org/80cc/cf9e43cadbb8390ec5f84b08fb8daa5d803e.pdf

United Nations Educational, Scientific and Cultural Organization, Unesco. (2005). Guidelines for inclusion: Ensuring access to education for all. Paris: Autor.

Verger, A., Bonal, X., y Zancajo, A. (2016). Recontextualización de políticas y (cuasi) mercados educativos. Un análisis de las dinámicas de demanda y oferta escolar en Chile. Education Policy Analysis Archives, 24, 27. https://doi.org/10.14507/epaa.24.2098 
Villalobos, C. y Valenzuela, J. P. (2012). Polarización y cohesión social del sistema escolar chileno. Revista de Análisis Económico, 27(2), 145-172. https://doi.org/10.4067/s0718-88702012000200005

Villegas, M., Simón, C., y Echeita, G. (2014). La inclusión educativa desde la voz de madres de estudiantes con trastornos del espectro autista en una muestra chilena. Revista Española de Discapacidad, 2(2), 63-82. https://doi.org/10.5569/2340-5104.02.02.04

Weinstein, J. \& Muñoz, G. (2014). What do we know about school principals? Lessons from the case of Chile. Interamerican dialogue. Recuperado de https://prealblog.files.wordpress.com/2014/12/final-school-principalsin-chile-english-fs-12-1-14.pdf

Weinstein, J., Cuellar, C., Hernández, M., y Fernández, M. (2016). Director(a) por primera vez. Un estudio sobre la experiencia y socialización de los directores noveles en establecimientos municipales en Chile. Revista Calidad en la Educación, 44, 12-45.

https://doi.org/10.4067/s0718-45652016000100002

Recibido: 15/03/2018

Aceptado: 21/09/2018 